\title{
Unitary Hyperperfect Numbers
}

\author{
By Peter Hagis, Jr.
}

\begin{abstract}
Unitary hyperperfect numbers are generalized unitary perfect numbers. In this paper a list of such numbers is given, and some results concerning them are proved.
\end{abstract}

1. Introduction. In this paper, lower-case letters denote positive integers, with $p$ and $q$ always representing primes. If $d \mid n$ and $(d, n / d)=1, d$ is said to be a unitary divisor of $n$. If $\sigma^{*}(n)$ denotes the sum of the unitary divisors of $n$, then $\sigma^{*}(1)=1$ while

$$
\sigma^{*}(n)=\left(1+p_{1}^{a_{1}}\right)\left(1+p_{2}^{a_{2}}\right) \cdots\left(1+p_{s}^{a_{3}}\right)
$$

if $n=p_{1}^{a_{1}} p_{2}^{a_{2}} \cdots p_{s}^{a_{s}}$. It is easy to see that $\sigma^{*}(n)=\sigma(n)$ (where $\sigma(n)$ denotes the sum of all the positive divisors of $n$ ) if and only if $n$ is square-free.

Subbarao and Warren [2] have defined $n$ to be a unitary perfect number if $\sigma^{*}(n)=2 n$. Minoli [1] has defined $m$ to be a hyperperfect number if $m=1+$ $t[\sigma(m)-m-1]$. We shall say that $m$ is a unitary hyperperfect number if

$$
m=1+t\left[\sigma^{*}(m)-m-1\right] \text {. }
$$

When $t=1, m$ is a unitary perfect number. A square-free number is hyperperfect if and only if it is unitary hyperperfect.

2. Some Elementary Results. Suppose that $m$ is a unitary hyperperfect number. From (2) it is immediate that $(m, t)=1$. Therefore, if $3 \mid t$, then $3 \nmid m$. If $t \equiv-1,1$ $(\bmod 3)$, then, from $(2), \sigma^{*}(m) \equiv 2,2 m(\bmod 3)$. Now, assume that $m=\prod_{j=1}^{r} p_{j}^{a_{j}}$ where $p_{j} \neq 3$. From $(1), \sigma^{*}(m)=\Pi_{j=1}^{r}\left(1+p_{j}^{a_{j}}\right)$ and since $\sigma^{*}(m) \neq 0(\bmod 3)$ it follows that $2 \mid a_{j}$ if $p_{j} \equiv-1(\bmod 3)$ and that $\sigma^{*}(m) \equiv 2^{r}(\bmod 3)$. Therefore, $m \equiv 1(\bmod 3)$ and $r$ is odd. We have proved:

FACT 1 . If $m$ is a unitary hyperperfect number and $3 \nmid m$, then $m \equiv 1(\bmod 3)$. Also, if $3 \nmid m t$, then $m$ has an odd number of distinct prime factors.

Since $\sigma^{*}\left(p^{a}\right)=p^{a}+1$, we have immediately from (2):

FACT 2. If $m$ is a unitary hyperperfect number, then $m \neq p^{a}$.

Now, suppose that $m=p^{a} q^{b}$ is a unitary $t$ hyperperfect number. Since $\sigma^{*}(m)=$ $m+p^{a}+q^{b}+1$, it follows easily from (2) that

$$
q^{b}=\left(t p^{a}+1\right) /\left(p^{a}-t\right),
$$

and we see immediately that $p^{a}>t$. If $p^{a}-t=1$, then

$$
q^{b}=p^{2 a}-p^{a}+1
$$

Received June 27, 1980.

1980 Mathematics Subject Classification. Primary 10A20. 
A search was made for all solutions of (4) with $2 \leqslant p^{a}<10^{5}$. There were 720. In 695 solutions, $a=b=1$. Eighteen solutions were of the form $p^{2} q$. The "exceptional" cases were $2^{4} \cdot 241,3^{4} \cdot 6481,3^{6} \cdot 530713,5^{4} \cdot 390061,7^{3} \cdot 117307,13^{4}$. 815702161 , and $19 \cdot 7^{3}$.

If, in (3), $p^{a}-t=2$, then

$$
2 q^{b}=\left(p^{a}-1\right)^{2}
$$

so that $p^{a}=2^{k}+1$. If $a=1$, then $p$ is a Fermat prime; if $a>1$, then $a=2$, $p=3, k=3$. Therefore, the only known unitary $t$ hyperperfect numbers of the form $p^{a} q^{b}$ with $p^{a}-t=2$ are $3 \cdot 2,5 \cdot 2^{3}, 17 \cdot 2^{7}, 257 \cdot 2^{15}, 65537 \cdot 2^{31}$ and $3^{2} \cdot 2^{5}$.

If $p^{a}-t=g$ in (3), then it is easy to see that $p^{2 a} \equiv-1(\bmod g)$. It follows that $4 \nmid g$ and that $g$ has no prime factors of the form $4 j+3$ since -1 is a quadratic nonresidue of all such primes. We have established:

FACT 3. If $m$ is a unitary $t$ hyperperfect number of the form $p^{a} q^{b}$, then $p^{a}-t=1,2,5,10,13,17,25,26,29,34, \cdots$.

If $m=p^{a} q^{b} r^{c}$ is a unitary $t$ hyperperfect number, then it follows from (2) (see also [3]) that

$$
\begin{gathered}
{\left[\left(p^{a}-t\right) q^{b}-t\left(p^{a}+1\right)\right]\left[\left(p^{a}-t\right) r^{c}-t\left(p^{a}+1\right)\right]} \\
=\left(p^{a}-t\right)\left(1+t p^{a}\right)+t^{2}\left(p^{a}+1\right)^{2}
\end{gathered}
$$

If $A B$, where $A<B$, is a factorization into positive integers of the right-hand member of (6), then it follows that

$$
q^{b}=\left[t\left(p^{a}+1\right)+A\right] /\left(p^{a}-t\right), \quad r^{c}=\left[t\left(p^{a}+1\right)+B\right] /\left(p^{a}-t\right)
$$

if the right-hand members of (7) are powers of distinct primes. Now suppose that $p^{a}-t=1$. Then

$$
q^{b}=p^{2 a}-1+A, \quad r^{c}=p^{2 a}-1+B, \quad A B=p^{4 a}-p^{2 a}-p^{a}+2
$$

If $A=1$, then $q^{b}=p^{2 a}$ which is impossible. A search was made for all solutions of (8) with $2 \leqslant p^{a} \leqslant 313$. Eight were found. For five of these $a=b=c=1$ so that $m$ is square-free and therefore hyperperfect (as well as unitary hyperperfect). These hyperperfect numbers were found earlier by te Riele and are given in [3]. The other three solutions yielded the unitary hyperperfect numbers $2 \cdot 5 \cdot 3^{2}, 5 \cdot 3^{3} \cdot 223$ and $11^{2} \cdot 14683 \cdot 4999387$.

3. The Unitary Hyperperfect Numbers Less Than $10^{6}$. Using the CDC Cyber 174 at the Temple University Computing Center, a search was made for all unitary hyperperfect numbers $m$ such that $m \leqslant 10^{6}$. The search required about one hour and fifteen minutes of computer time, and 36 numbers were found. Four were unitary perfect numbers and were found by Subbarao and Warren [2]. Twenty of the others were square-free and appear in the list of hyperperfect numbers given by 
Minoli [1]. The twelve new ones are: $2^{3} \cdot 5(t=3) ; 2^{2} \cdot 13(t=3) ; 2^{5} \cdot 3^{2}(t=7)$; $3^{2} \cdot 73(t=8) ; 2^{7} \cdot 17(t=15) ; 2^{4} \cdot 241(t=15) ; 2^{5} \cdot 173(t=27) ; 7^{3} \cdot 19(t=18) ;$ $5^{2} \cdot 601(t=24) ; 3^{3} \cdot 5 \cdot 223(t=4) ; 7^{3} \cdot 307(t=162) ; 3^{4} \cdot 6481(t=80)$.

Department of Mathematics

Temple University

Philadelphia, Pennsylvania 19122

1. D. MinoLI, "Issues in nonlinear hyperperfect numbers," Math. Comp., v. 34, 1980, pp. 639-645.

2. M. V. Subbarao \& L. J. Warren, "Unitary perfect numbers," Canad. Math. Bull., v. 9, 1966, pp. 147-153.

3. H. J. J. TE RIRLE, "Hyperperfect numbers with three different prime factors," Math. Comp., v. 36, 1981, pp. 297-298. 